\title{
Tuning the water reduction through controlled nanoconfinement within an organic liquid matrix
}

Nicolas Dubouis ${ }^{1,2,5}$, Alessandra Serva ${ }^{3,5}$, Roxanne Berthin ${ }^{3,5}$, Guillaume Jeanmairet ${ }^{3,5}$, Benjamin Porcheron ${ }^{4,5}$, Elodie Salager ${ }^{4,5}$, Mathieu Salanne $e^{3,5^{*}}$, Alexis Grimaud $d^{1,2,5^{*}}$

1. Chimie du Solide et de l'Energie, Collège de France, UMR 8260, 75231 Paris Cedex 05

2. Sorbonne Université, Paris, France

3. Sorbonne Université, CNRS, Physico-Chimie des Électrolytes et Nanosystèmes Interfaciaux, F-75005 Paris, France

4. CNRS-CEMHTI, UPR3079, Université d'Orléans, 1D avenue de la recherche scientifique, 45071 Orléans Cedex, France

5. Réseau sur le Stockage Electrochimique de l'Energie (RS2E), CNRS FR3459, 33 rue Saint Leu, 80039

Amiens Cedex, France

*Corresponding authors:

Alexis Grimaud : alexis.grimaud@college-de-france.fr

Mathieu Salanne: mathieu.salanne@sorbonne-universite.fr 


\begin{abstract}
The growing hydrogen-economy requires accelerating the hydrogen evolution reaction. The water dissociation step (Volmer step) has been proposed as a main kinetic limitation, but the mechanisms at play in the electrochemical double-layer are poorly understood. This is due to the ambivalent role of water: it acts both as a reactant and as a solvent. Here we propose to confine water inside an organic liquid matrix in order to isolate the sole role of water as a reactant. We observe the formation of aqueous-rich nanodomains, which size can be tuned by changing the supporting electrolyte, and found that the reactivity of the system significantly varies with its nanostructure. Depending on the conditions, it is dominated by either the strength of short-range cation-water interactions or the formation of long chains of water molecules. This understanding paves the way toward the development of more efficient and selective electrocatalysts for the water, $\mathrm{CO}_{2}, \mathrm{O}_{2}$ or $\mathrm{N}_{2}$ reduction.
\end{abstract}




\section{Introduction}

The conversion of electricity into chemical fuels is of prime interest to overcome the intermittency of renewable energies that hampers their massive deployment. Thanks to its versatility, hydrogen fuel $\left(\mathrm{H}_{2}\right)$ is expected to be one of the big players for the global decarbonization since it can be used to power zero emission vehicles, be transformed into electricity, and feed the chemical industry. While seductive, the hydrogen production through water electrolysis (water splitting: $\mathrm{H}_{2} \mathrm{O}=\mathrm{H}_{2}+1 / 2 \mathrm{O}_{2}$ ) suffers from several fundamental challenges that have to be tackled before it can become cost-competitive against hydrogen generated by reforming of fossil fuels. Because the anodic oxygen evolution reaction $\left(\mathrm{OER}: 2 \mathrm{H}_{2} \mathrm{O}=\mathrm{O}_{2}+4 \mathrm{H}^{+}\right.$ $\left.+4 \mathrm{e}^{-}\right)$exhibits large overpotentials compared to the one of the hydrogen evolution reaction ( $\mathrm{HER}: 2 \mathrm{H}^{+}+2 \mathrm{e}^{-}$ $=\mathrm{H}_{2}$ ) in acidic media, it has attracted most of the efforts, in particular for improving the performances and the stability of Ir-based catalysts. ${ }^{2}$ Nevertheless, the foreseen development of alkaline anion exchange membrane water electrolyzers ${ }^{2}$ recently shifted the attention on the sluggish kinetics of the HER in alkaline media when compared to acidic conditions. ${ }^{3-6}$ Understanding the physical origin of these slow kinetics for the HER in alkaline electrolytes has now become a main objective of the electrocatalysis community.

Traditionally, the HER performances of different catalysts is explained by the use of descriptors approximating the energy of the reaction intermediates with physical in vacuum parameters intrinsic of the surface, such as the hydrogen binding energy (HBE) on the surface of the catalyst. ${ }^{7,8}$ Nevertheless, despite some attempts, ${ }^{9,10}$ those intrinsic properties fail to capture the influence of extrinsic parameters such as the $\mathrm{pH}^{11,12}$ or the presence of $\mathrm{Li}^{+}$cations in the electrolyte, ${ }^{6}$ on the HER activity. ${ }^{13}$ These limitations urged the need for understanding the water environment within the electrochemical double-layer (EDL) and its impact on the reactivity of water. Hence, the use of operando spectroscopies such as X-Ray absorption spectroscopy ${ }^{14,15}$ (XAS) or Raman spectroscopy ${ }^{16}$ coupled with ab-initio calculations revealed a strong dependence of the interfacial $\mathrm{H}$-bond network with the applied potential. Furthermore, a model involving an increased rigidity of this network in alkaline electrolyte, which would hinder the transfer of water ions 
$\left(\mathrm{H}_{3} \mathrm{O}^{+}\right.$or $\left.\mathrm{OH}^{-}\right)$across the $\mathrm{EDL}$, was recently proposed. This model, supported by laser temperature-jump measurements, provides an interesting lead for the slow kinetics measured for the HER in alkaline electrolytes. ${ }^{17,18}$ Nevertheless, our understanding of the impact of weak interactions in the electrolyte on the HER kinetics remains elusive at the molecular level.

a

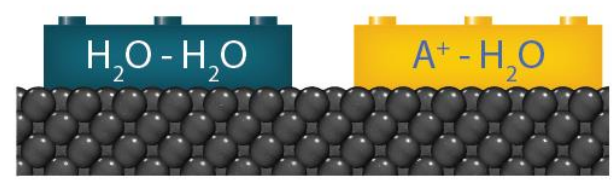

b

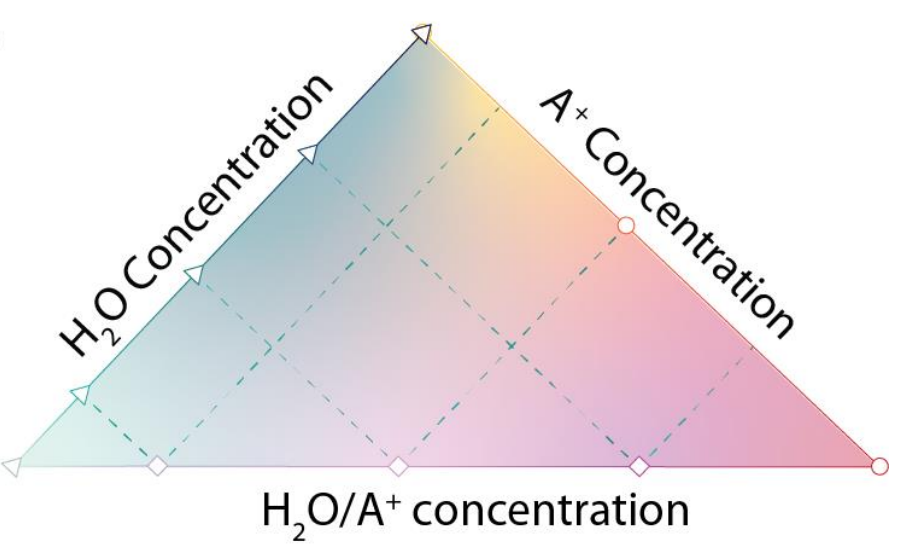

Figure 1. Chemical strategy to select long- and short-range interactions. a) Building blocks representing the interactions between water-water (blue) or cation-water (yellow) molecules that are studied and b) visual representation of the 3 series of systems that can be studied by playing on the water or supporting salt concentration.

In order to draw this molecular understanding for the dependence of the HER on the electrolyte composition/structure, one must first define a strategy to decouple the effect of each interaction on the reaction kinetics. Indeed, while at short-range, non-covalent interactions between water molecules $\left(\mathrm{H}_{2} \mathrm{O}\right.$ $\mathrm{H}_{2} \mathrm{O}$ ) or between water and the supporting-salt cation $\left(\mathrm{A}^{+}-\mathrm{H}_{2} \mathrm{O}\right)$ are competing (Figure 1.a), at long-range the formation of a hydrogen bonding network and its dynamics must also be considered. Here we apply a chemical strategy consisting in constraining the water environment by diluting it in a non-reactive matrix, i.e. in an organic solvent (acetonitrile), stable at the working potentials, in order to form nanoscale reactors.19,20 This strategy allows us to confine water in a situation where it no longer plays the dual role of solvent and reactant. We select the interactions of interest by controlling independently or concomitantly the water and salt concentrations (as well as the nature of the cation), as schematized on Figure 1.b. The reactivity of water is assessed by electrochemical measurements, while the structure of the systems is 
analyzed by combining classical molecular dynamics (MD) simulations (at the bulk or interface levels) with nuclear magnetic resonance (NMR) spectroscopy analysis that provide critical information regarding the acidity of the protons, the strength and the amount of $\mathrm{H}$-bonds and small-angle $\mathrm{X}$-ray scattering, which gives us an information on the structure at medium to long-ranges. Doing so, we draw a water structure/reactivity model that depends on two main parameters: 1) the nature of the cation and 2) the size of the nanodomains formed by the water molecules and the salt inside the matrix. More specifically, our study highlights that mastering short-range and long-range noncovalent interactions can help to tune the water reactivity at electrified interfaces, which is at the heart of a wide variety of electrochemical devices.

\section{Cation-water vs. water-water interactions}

a

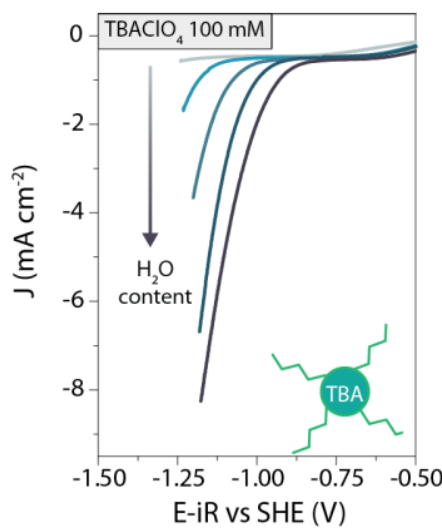

b

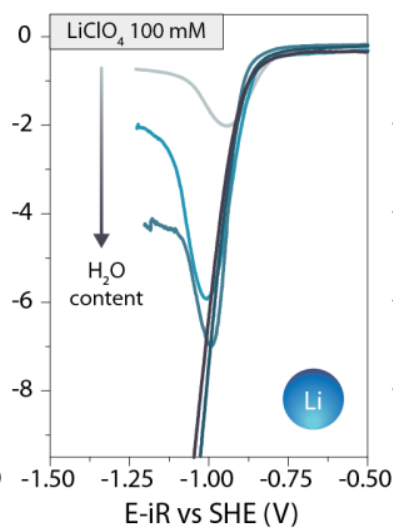

C

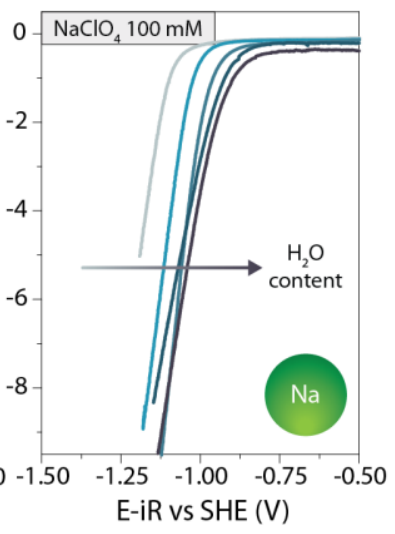

d

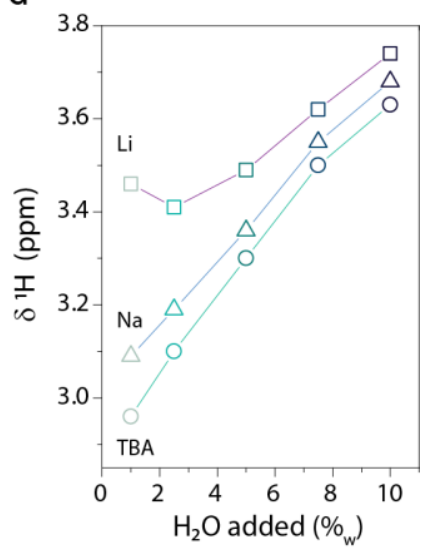

Figure 2. Modification of the reactivity of water depending on its environment. Linear sweep voltammograms recorded in acetonitrile in the presence of a) $100 \mathrm{mM}$ of $\mathrm{TBAClO}_{4}$ b) $100 \mathrm{mM}$ of $\mathrm{LiClO}_{4}$ and c) $100 \mathrm{mM}$ of NaClO 4 with different water contents $(1$, 2.5, 5, 7.5 and $10 \%$ in mass from light to dark blue, the exact values are given in the Supplementary Tables 1 and 2) on a rotating disk (1 $600 \mathrm{rpm}$ ) Pt electrode with a $50 \mathrm{mV} \mathrm{s}^{-1}$ sweeping rate. d) Evolution of the ${ }^{1} \mathrm{H}$ chemical shift of the water molecules in these electrolytes as a function of the water content.

Among the different interactions playing a role on the water reactivity, our attention first focused on the short-range $\mathrm{A}^{+}-\mathrm{H}_{2} \mathrm{O}$ and $\mathrm{H}_{2} \mathrm{O}-\mathrm{H}_{2} \mathrm{O}$ interactions and their competing effects. In order to compare the relative weight of these interactions on the reactivity of water at the electrochemical interfaces, we studied by rotating disk electrode $(\mathrm{RDE})$ using a platinum electrode different supporting salts, i.e. tetrabutylammonium 
perchlorate $\left(\mathrm{TBAClO}_{4}\right)$, sodium perchlorate $\left(\mathrm{NaClO}_{4}\right)$ or lithium perchlorate $(\mathrm{LiClO})$ in acetonitrile $(\mathrm{ACN})$ at increasing water concentration from 1\% (4.4 molecules of water per cation) to 10\% (44 molecules of water per cation) in mass. When using $\mathrm{TBAClO}_{4}$ as a supporting electrolyte, no water reduction is measured at low water concentration (Figure 2.a), which can be explained by the formation of a TBA+-rich EDL. Indeed, since the water molecules hardly enter the solvation shell of $\mathrm{TBA}^{+}$cations, their presence in the EDL under negative polarization prevents water to access the interface and therefore to be reduced. ${ }^{19-22}$ Nevertheless, by increasing the concentration of water in the organic electrolytes, water reduction slowly takes off with its onset potential shifting toward less negative values at higher water contents. Unlike for $\mathrm{TBAClO}_{4}$, when using $\mathrm{LiClO}_{4}$ as supporting salt, water reduction is measured even at a water concentration as low as $1 \%$ (Figure 2.b). Furthermore, the onset potential is not altered by the water concentration. Indeed, increasing the water concentration only gradually transforms the reduction event from a peak which arises from the passivation of the electrode due to the precipitation of $\mathrm{LiOH}$ at low water content, ${ }^{19}$ to a classical exponential event as expected for the catalytically activated HER. Such precipitation phenomenon is simply explained first by the formation of $\mathrm{OH}^{-}$following the HER and second by the greatest solubility of $\mathrm{LiOH}$ in water than in pure acetonitrile, leading to an absence of the electrode passivation at greater water content. Finally, when the cation is changed to sodium $\left(\mathrm{Na}^{+}\right)$, the reduction of water is observed even at low water concentration, but at more negative potential than with $\mathrm{Li}^{+}$cations (Figure 2.c). Furthermore, alike for $\mathrm{TBA}^{+}$cations, the increase of the water content in the electrolyte shifts the onset potential for $\mathrm{H}_{2} \mathrm{O}$ reduction toward less negative potentials. While this shift observed for $\mathrm{Na}^{+}$and $\mathrm{TBA}^{+}$suggests that $\mathrm{H}_{2} \mathrm{O}-\mathrm{H}_{2} \mathrm{O}$ interactions that presumably increase at greater water concentration are likely to influence the HER within these electrolytes, the different behaviors measured in the presence of these three different cations confirms a competing effect between $\mathrm{H}_{2} \mathrm{O}-\mathrm{H}_{2} \mathrm{O}$ and $\mathrm{A}^{+}-\mathrm{H}_{2} \mathrm{O}$ interactions.

To gain further understanding into this competition, insights on the chemical environment of protons from water molecules is obtained by the means of NMR spectroscopy. At $1 \%$ water concentration, the ${ }^{1} \mathrm{H}$ chemical shift of $\mathrm{H}_{2} \mathrm{O}$ molecules follows the same order as the onset potential for water reduction: $\mathrm{Li}^{+}>\mathrm{Na}^{+}>\mathrm{TBA}^{+}$ 
(Figure 2.d). Since a more positive chemical shift reflects a weaker electronic density, this trend demonstrates that the strength of the $\mathrm{A}^{+}-\mathrm{H}_{2} \mathrm{O}$ interaction is correlated with the Lewis acidity of the cation following the order $\mathrm{Li}^{+}>\mathrm{Na}^{+}>\mathrm{TBA}^{+}$which results in a weaker $\mathrm{O}-\mathrm{H}$ bond and easier proton adsorption (the so called Volmer step: $\left.\mathrm{H}_{2} \mathrm{O}+\mathrm{e}^{-}=\mathrm{H}_{\mathrm{ads}}+\mathrm{OH}^{-}\right){ }^{19}$ This is coherent with a recent ab-initio MD simulation study of aqueous solutions of alkali metal salts under applied electric field. ${ }^{23}$ It showed that the water dissociation threshold field is lowered in $\mathrm{LiCl}$ and $\mathrm{NaCl}$ with respect to bulk water (with an effect slightly more marked in the case of the lithium salt), but not in the less acidic $\mathrm{KCl}$. For electrolytes using $\mathrm{TBA}^{+}$and $\mathrm{Na}^{+}$cations, a monotonous increase of the proton chemical shift with the water concentration is measured (Figure 2.d). Monitoring that the shift of the peak related to the $\mathrm{CH}_{3}$ group in $\mathrm{ACN}$ is almost unaffected, we can discard changes coming from a drastic modification of the electrolytes magnetic susceptibility as the origin for this shift (Figure S1). Thus, this downfield shift toward values closer of a bulk $\mathrm{H}_{2} \mathrm{O}$ environment reflects a greater acidity for protons that matches well with their increased reactivity. In contrast, in the presence of $\mathrm{LiClO}_{4}$, a "V-shaped" curve characterizes the evolution of the ${ }^{1} \mathrm{H}$ chemical shift for protons in water with its concentration (Figure 2.d). Surprising at the first glance, such a behavior can be attributed to the existence of different water populations with an exchange rate high enough to observe the coalescence of their respective NMR signals. ${ }^{24}$ This existence of different $\mathrm{H}_{2} \mathrm{O}$ populations in the presence of $\mathrm{Li}^{+}$cations would confirm a stronger $\mathrm{A}^{+}-\mathrm{H}_{2} \mathrm{O}$ interaction in the latter case. Finally, it is noteworthy that changing the anion from $\mathrm{ClO}_{4}^{-}$to TFSl is not altering the aforementioned experimental trends (Figure S2), confirming that in these conditions the effect of water-water and water-cations interactions prevail on the reactivity of water. Nevertheless, while NMR reveals a drastic influence of the water concentration on the protons environment, it fails to explain why an increase of the chemical shift for the Li+ electrolytes does not lead to more facile water reduction. 

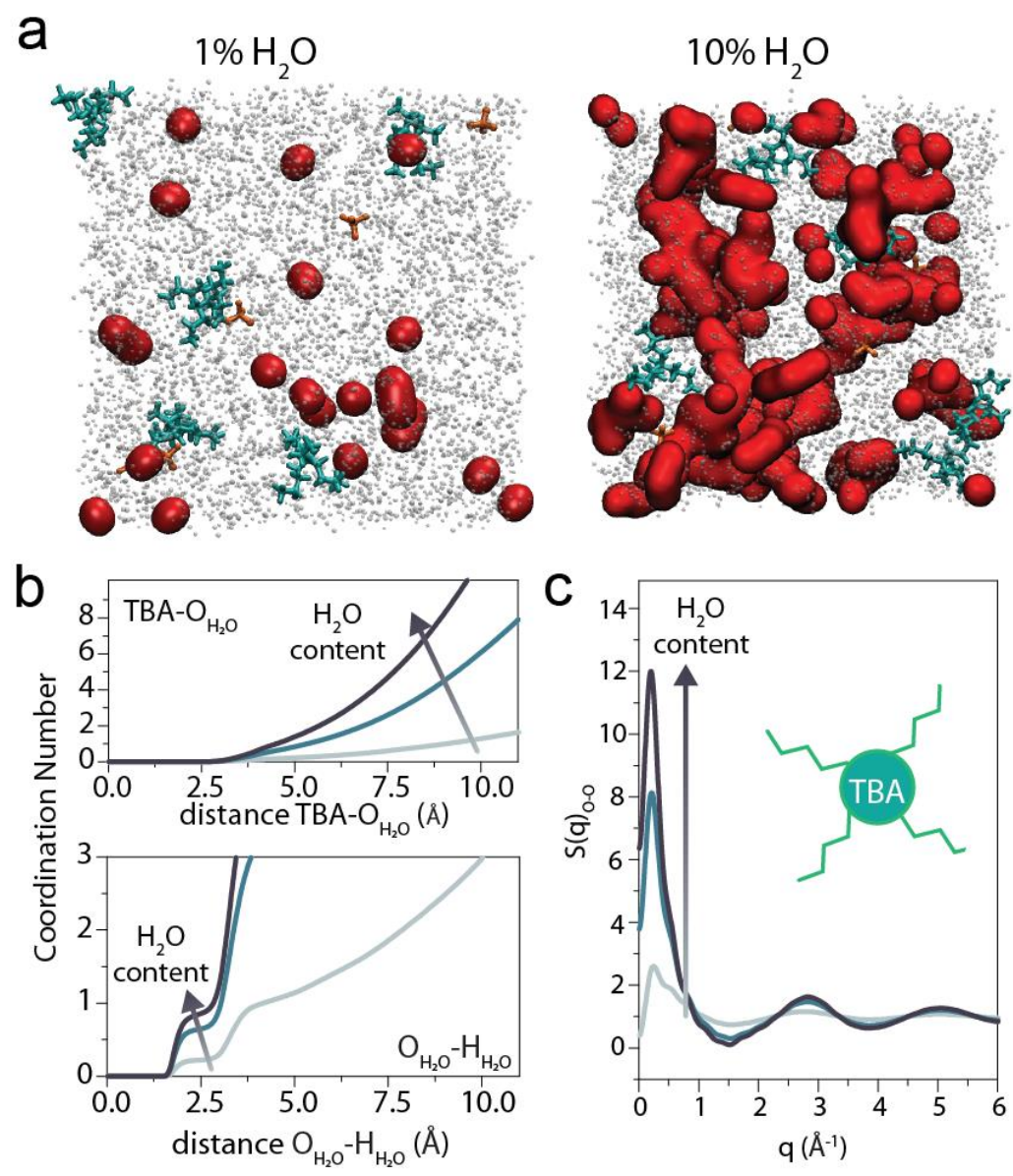

Figure 3. Water structure in the vicinity of hydrophobic TBA+ cation. a) MD snapshots for the systems containing 1\% and $10 \%$ of water in mass in the presence of $100 \mathrm{mM} \mathrm{TBAClO}_{4}$ in acetonitrile. Water molecules are represented in red, $\mathrm{TBA}^{+}$cations in green, $\mathrm{ClO}_{4}^{-}$anions in orange and acetonitrile solvent with grey points. b) Coordination numbers for TBA+- $\mathrm{H}_{2} \mathrm{O}$ and $\mathrm{H}_{2} \mathrm{O}-\mathrm{H}_{2} \mathrm{O}$ and c) structure factor for the oxygen atom of $\mathrm{H}_{2} \mathrm{O}$ molecules at different water concentrations (1\% in grey, $5 \%$ in blue, $10 \%$ in dark blue).

Additional understanding on the molecular environment of water molecules in these different systems was thus gleaned performing classical MD simulations of the bulk electrolytes. To no surprise, for the $\mathrm{TBAClO}_{4}$ electrolytes, the water environment is found drastically different at $1 \%$ - isolated $\mathrm{H}_{2} \mathrm{O}$ molecules - from the one observed at $10 \%$ where the formation of nanodomains of clustered water molecules can be found, as illustrated on the snapshots in Figure 3.a. Furthermore, the coordination numbers between $\mathrm{TBA}^{+}$cations and water molecules (Figure 3.b, top) at short distances only weakly depends on water concentration, confirming the absence of favorable interactions between $\mathrm{H}_{2} \mathrm{O}$ and TBA+ cations (as illustrated by the broadness of the first peak in the radial distribution functions, Figure S3). The rise of the coordination number between the 
water molecules (Figure 3.b, bottom) with the water concentration pinpoints an increase of H-bond interactions between the water molecules at high water concentration. This increasing amount of interactions is confirmed by the growth of a peak at low-q in the computed $\mathrm{O}_{\text {water }}-\mathrm{O}_{\text {water }}$ structure factor (Figure 3.c) indicative of a long-range organization for water molecules and the formation of water clusters that results from the increase of $\mathrm{H}$-bonds between water molecules. Thus, the average environment of the water molecules becomes more similar to a bulk water environment, which explains the downfield in the NMR ${ }^{1} H$ chemical shift (Figure 2.d). Here again, the more facile water reduction measured when nanodomains start to be formed may be explained using previous ab-initio MD results. Indeed, early studies highlighted the need for a favorable $\mathrm{H}$-bonding environment for the transfer of both the proton ${ }^{25}$ and the hydroxide ${ }^{26}$ ions. As a consequence, when autoprotolysis reaction occurs, the transfer of protons along a hydrogen bond "wire" allows the two ionic products to be separated by three or more neighbors. If this wire remains unbroken, the ions recombine rapidly. ${ }^{27} \mathrm{~A}$ similar picture arised from a static density functional theory (DFT) study of the palladium-water interface. ${ }^{28}$ Here we can draw an analogy and hypothesize that larger clusters facilitate the separation of the product of the Volmer step in the $\mathrm{TBAClO}_{4}$ electrolyte. 

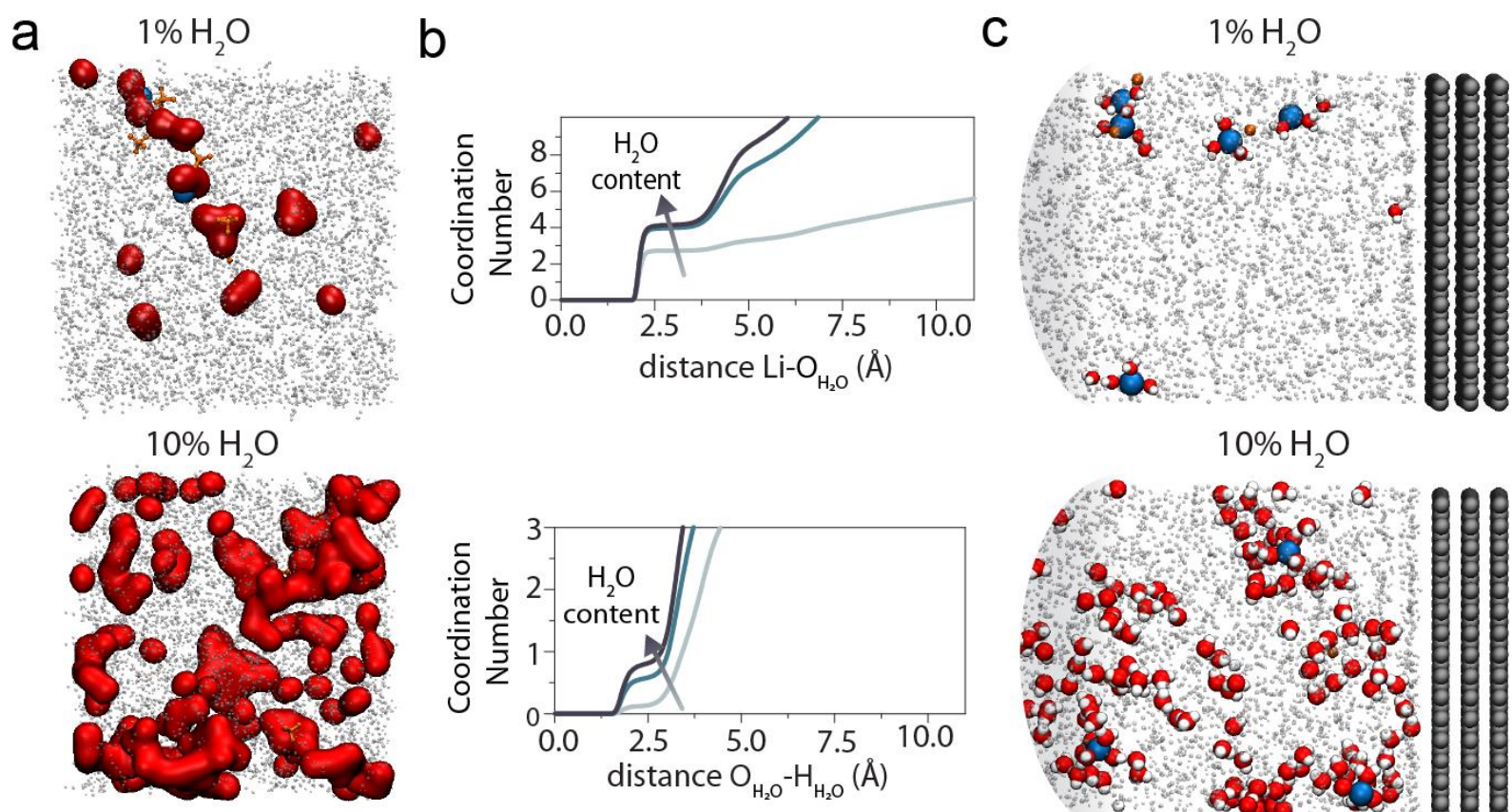

$10 \% \mathrm{H}_{2} \mathrm{O}$

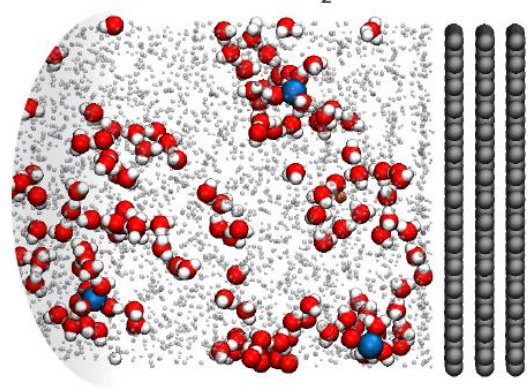

Figure 4 . Formation of $\mathrm{Li}^{+}-\mathrm{H}_{2} \mathrm{O}$ nanoscale reactors. a) MD snapshots for the systems containing $1 \%$ and $10 \%$ of water in mass in the presence of $100 \mathrm{mM} \mathrm{LiClO}_{4}$ in acetonitrile. Water molecules are represented in red, $\mathrm{Li}^{+}$cations in blue, $\mathrm{ClO}_{4}^{-}$anions in orange and acetonitrile solvent with grey points. b) Coordination numbers for $\mathrm{Li}^{+}-\mathrm{H}_{2} \mathrm{O}$ and $\mathrm{H}_{2} \mathrm{O}-\mathrm{H}_{2} \mathrm{O}$ and c) MD snapshots of the double-layer structure at the negative electrode for $1 \%$ (top) or 10\% (bottom) of water in mass.

Puzzled by the different experimental trends observed for the $\mathrm{LiClO}_{4}$ containing electrolytes, we also analyzed the water environment with MD simulations for the Li+-containing systems. As previously shown, ${ }^{19}$ at low water concentration ( $1 \%$ in mass $~ 400 \mathrm{mM}$ ), water molecules are found to preferentially coordinate the $\mathrm{Li}^{+}$cations (Figure 4.a top). Unlike for $\mathrm{TBA}^{+}$cations that are hydrophobic, water is found to replace acetonitrile and perchlorate anions (Figure S4) in the first solvation shell of $\mathrm{Li}^{+}$cations (Figure 4.b). This is further confirmed by observing a downfield shift of the ${ }^{7}$ Li chemical shift in these electrolytes to values close to those observed in aqueous electrolytes (Figure S5). Thus, at intermediate water contents, water is either coordinating $\mathrm{Li}^{+}$cations (unshielded environment) or free in the electrolyte (shielded environment) (Figure 2.d). Alike for $\mathrm{TBAClO}_{4}$, at higher water content, water clusters are forming in the electrolyte (Figure 4.a bottom) with the increase of the coordination between water molecules (Figure 4.b) being responsible for this aggregation. Thus, the increase of $\mathrm{H}_{2} \mathrm{O}-\mathrm{H}_{2} \mathrm{O}$ interactions explains the downfield observed in the NMR 
spectra at higher water content. The MD simulations of the bulk of the electrolyte also reveals that the energy required to extract one water molecule from the $\mathrm{Li}^{+}$cation solvation shell ( $200 \mathrm{meV}$ ) is greater when compared to the energy required to separate two water molecules ( $80 \mathrm{meV}$ ) (Figure S6), and that the strength of these weak $\mathrm{H}_{2} \mathrm{O}-\mathrm{H}_{2} \mathrm{O}$ interactions is weakly affected by the water content. Hence, it confirms that the short-range $\mathrm{H}_{2} \mathrm{O}-\mathrm{H}_{2} \mathrm{O}$ interactions are much weaker than $\mathrm{Li}^{+}-\mathrm{H}_{2} \mathrm{O}$ interactions, independently of the $\mathrm{H}_{2} \mathrm{O}$ content in the electrolyte. In order to confirm that the different water environments isolated in the bulk of the organic electrolyte are maintained at the interface, simulations were carried out applying a constant voltage $(1 \mathrm{~V})$ between two planar carbon electrodes to investigate the water structure within the EDL. Interestingly, the water organization is found to be similar at the negative electrode as in the bulk of the electrolyte. Hence, water molecules are found to coordinate $\mathrm{Li}^{+}$cations in both systems (Figure 4.c), and some clusters of non-coordinating water are present in the water-rich electrode, surrounding the $\mathrm{Li}^{+}-\left(\mathrm{H}_{2} \mathrm{O}\right)_{\times}$ adducts. This undoubtedly establishes that the intensity of the short-range $\mathrm{Li}^{+}-\mathrm{H}_{2} \mathrm{O}$ prevails on $\mathrm{H}_{2} \mathrm{O}-\mathrm{H}_{2} \mathrm{O}$ interactions and dictates the water reactivity at the interface. Finally, the case of the sodium electrolyte is logically an intermediate between these two extreme cases. While the electrolyte structure is similar to the one of the lithium (Figure S7), the weaker interactions between water molecules and $\mathrm{Na}^{+}$cations when compared to $\mathrm{Li}^{+}$cations (Figure S8) explain why when increasing the $\mathrm{H}_{2} \mathrm{O}-\mathrm{H}_{2} \mathrm{O}$ interactions, the reactivity of water is found to increase.

\section{Enlarging the aqueous nanodomains to promote the water reactivity}

At this point of the study, we managed to tune the nature of the cation-water interaction, and to study how increasing the $\mathrm{H}_{2} \mathrm{O}-\mathrm{H}_{2} \mathrm{O}$ interactions is affecting the water reactivity. Nevertheless, while some long-range organization of water was found for the electrolytes with the highest water content, a direct correlation with water reduction could be drawn for the $\mathrm{TBA}^{+}$and the $\mathrm{Na}^{+}$-based electrolytes only. Hence, we then extended our study for Li+-based electrolytes by forming larger aqueous-rich domains to increase the long-range interactions within the electrolyte. While such nanodomains were already reported for water into acetonitrile 
at large $\mathrm{H}_{2} \mathrm{O}$ content, ${ }_{1}^{29-32}$ their formation is triggered (and enhanced) here by the addition of a salt at large concentration. $^{33}$

a

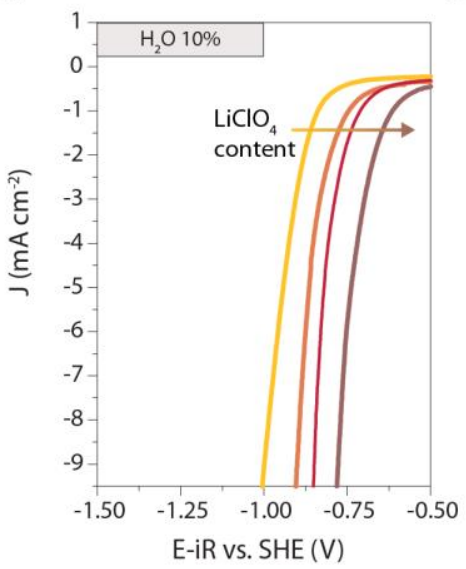

b

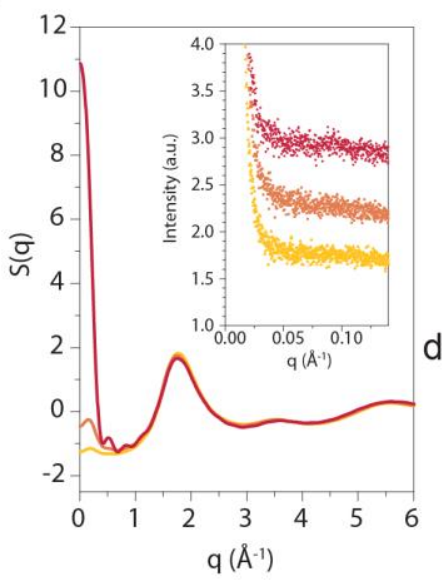

C
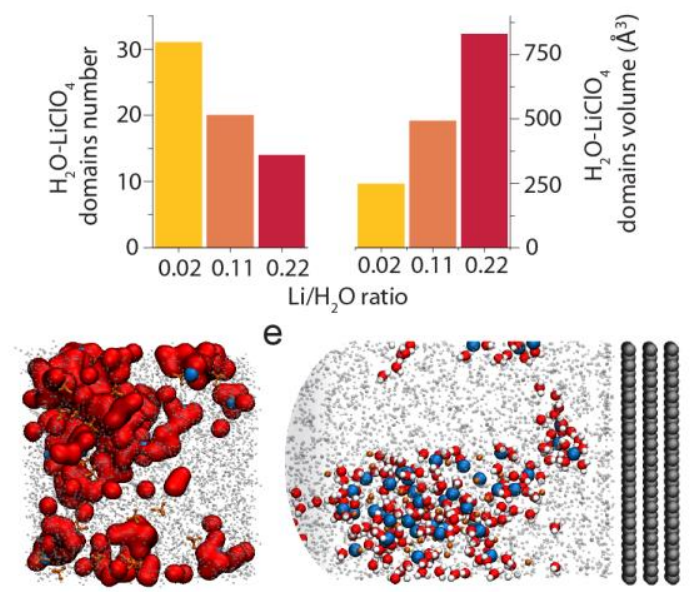

Figure 5 Formation of nanochannels to enhance the water reduction. a) Linear sweep voltammograms recorded in acetonitrile with $10 \%$ in mass of added $\mathrm{H}_{2} \mathrm{O}$ in the presence of $100 \mathrm{mM}$ (yellow), $500 \mathrm{mM}$ (orange), $1 \mathrm{M}$ (red), or $2 \mathrm{M}$ (brown) $\mathrm{LiClO}_{4}$ on a rotating disk (1 $600 \mathrm{rpm}$ ) Pt electrode with a $50 \mathrm{mV} \mathrm{s}^{-1}$ sweeping rate. b) Total X-ray-weighted structure factor of the electrolytes containing $10 \% \mathrm{H}_{2} \mathrm{O}, 100 \mathrm{mM}$ (yellow), $500 \mathrm{mM}$ (orange), $1 \mathrm{M}$ (red) $\mathrm{LiClO}_{4}$ as computed from the MD simulations. The insert represents the SAXS intensity experimentally measured. c) Number of $\mathrm{LiClO}_{4} \mathrm{H}_{2} \mathrm{O}$ domains and their average volume depending on the $\mathrm{LiClO}_{4} / \mathrm{H}_{2} \mathrm{O}$ ratio at $10 \%$ in mass of water calculated from the MD simulations. d) MD snapshots of the concentrated electrolyte $\left(1 \mathrm{M} \mathrm{LiClO}_{4}\right.$ and $10 \%$ $\mathrm{H}_{2} \mathrm{O}$ ) showing the nanodomains in the bulk and e) at the cathodic interface.

The concentration of $\mathrm{LiClO}_{4}$ was thus gradually increased from $\sim 100 \mathrm{mM}$ to $\sim 2 \mathrm{M}$ in the $10 \% \mathrm{H} 2 \mathrm{O}$ in mass electrolytes (see Table S3 for exact values). As the $\mathrm{LiClO}_{4}$ concentration increases, the onset potential for water reduction is shifted toward less negative values (Figure 5.a), which is consistent with the increase in the water ${ }^{1} \mathrm{H}$ chemical shift measured by NMR (Figure S9). To better grasp the molecular origin for this enhanced reactivity, the electrolyte structure was analyzed with MD simulations and, at short-range, $\mathrm{Li}_{-} \mathrm{H}_{2} \mathrm{O}$ interactions were found to be gradually replaced by ion-pairing (Figure S10). These results are confirmed by the typical "bell-shape" curve obtained for the conductivity of these electrolytes as well as by the decrease of the ${ }^{7}$ Li chemical shift (Figure S11). 
The formation of water-rich domains at the nanoscale may result either in a fluctuation of the species concentration or in the microsegregation between these heterogeneities that can be experimentally accessed by small-angle X-ray scattering (SAXS) at the low-q values which reflects changes in the structure factor. ${ }^{32,34}$ The increase of the SAXS intensity at low-q values with the $\mathrm{LiClO}_{4}$ concentration reveals the growth of nanodomains (Figure 5.b, inset). This observation is corroborated by the increase of the X-ray-weighted structure factor calculated from the MD simulations at the low-q values (Figure 5.b) that arises from the formation of oxygen-rich domains (i.e. rich in $\mathrm{H}_{2} \mathrm{O}$ molecules and $\mathrm{ClO}_{4}^{-}$anions) (Figure S12). Hence, these results suggest that increasing the $\mathrm{LiClO}_{4}$ concentration enhances the size of the formed aqueous-rich nanodomains in the organic electrolyte, as visually illustrated by a MD snapshot of the electrolyte containing $1 \mathrm{M}$ of $\mathrm{LiClO}_{4}$ and $10 \%$ of water in mass (Figure 5.d). This interpretation is further reinforced by observing that while activation energies for the $\mathrm{Li}^{+}$and $\mathrm{H}_{2} \mathrm{O}$ diffusion measured by diffusion-NMR spectroscopy (Figure S13 and Supplementary Table 5) differ at low concentration $\left(E_{a, D_{L i}{ }^{+}}=42 \mathrm{~kJ} \cdot \mathrm{mol}^{-1}\right.$ and $E_{a, D_{\mathrm{H}_{2} O}}=$ $31 \mathrm{~kJ} \mathrm{~mol}^{-1}$ at $100 \mathrm{mM} \mathrm{LiClO}$ ), they become closer to each other when the $\mathrm{LiClO}_{4}$ concentration is increased to $1 \mathrm{M}\left(E_{a, D_{L i}}=32 \mathrm{~kJ} \cdot \mathrm{mol}^{-1}\right.$ and $\left.E_{a, D_{\mathrm{H}_{2} \mathrm{O}}}=28 \mathrm{~kJ} \cdot \mathrm{mol}^{-1}\right)$. As a result, we can assume that the diffusion of both $\mathrm{Li}^{+}$and $\mathrm{H}_{2} \mathrm{O}$ becomes correlated with the formation of these $\mathrm{H}_{2} \mathrm{O}-\mathrm{LiClO}_{4}$ rich nanodomains in the electrolyte. More quantitative information is obtained by performing a domain analysis ${ }^{35}$ : when $\mathrm{LiClO}_{4}$ concentration is increased from $100 \mathrm{mM}$ to $\sim 1 \mathrm{M}$ (at fixed water concentration), the number of $\mathrm{H}_{2} \mathrm{O}-\mathrm{LiClO}_{4}$ domains follows a two-fold decrease while their average volume increases by a factor 4 (Figure 5.c). Interestingly, when an electrical potential is applied, these large water-rich domains are conserved, as shown in Figure 5.e, and migrate to the negative electrode (Figure S14), forming aqueous-rich "channels" between the interface and the bulk. The presence of these large water-rich aggregates strikingly contrasts with our previous observations at low $\mathrm{LiClO}_{4}$ content for which only small water clusters could be found in the bulk and at the EDL. While these results suggest that the long-range ordering of the aqueous phase within the electrolyte is likely to impact the water reduction, the environment of water at short-range conspicuously diverges between the different electrolytes studied. Nevertheless when keeping the $\mathrm{H}_{2} \mathrm{O} / \mathrm{Li}^{+}$ratio constant, 
the third axis of the triangle in Figure 1, the short-range environment for $\mathrm{Li}^{+}$and $\mathrm{H}_{2} \mathrm{O}$ is not altered and a simultaneous increase of the $\mathrm{H}_{2} \mathrm{O}$ and $\mathrm{Li}^{+}$concentration does not modify the number of domains but rather increases their size (Figure S15). The presence of larger nanodomains drives the water reduction to higher potential (Figure S15), confirming the prime importance of the long-range interactions.

\section{Discussion}

Our combined experimental and theoretical observations evidence the crucial role of the long-range organization of water-rich domains on the water electrochemical activity. They provide a picture at the molecular scale of the complex role of the water at electrochemical interfaces. Notably, the greatest reactivity measured for water in large water-rich domains can be explained with the current knowledge of the bulk water autoprotolysis mechanism ${ }^{25,26}$ as well as the framework developed by Filhol et al. ${ }^{28}$ which suggests that several "layers" of water are required to accomplish the Volmer step: the first one provides proton to the electrode, the second accepts the $\mathrm{OH}^{-}$generated during the HER and the following ones allow the reactants and products to diffuse into solution following a Grotthuss diffusion mechanism. It is also noteworthy that a higher $\mathrm{Li}^{+}$concentration in the electrolyte may also drive the $\mathrm{OH}^{-}$transport across the double layer and promote the cleavage of the water $\mathrm{O}-\mathrm{H}$ bond, as previously suggested. ${ }^{23,36}$ From these experimental and modeling observations, we can therefore conclude that the highest activity measured for water reduction in presence of these nanodomains highlights the critical role of the long-range-interactions onto the water reactivity.

From both an experimental and a modeling point of view, we have highlighted in this work the critical role played by the organization of water at both short and long-ranges on its reactivity upon electrochemical reduction. By playing on the water content and the salt concentration/nature in the electrolytes, we could constrain the structure of water and sort out 4 different behaviors. When the $\mathrm{H}_{2} \mathrm{O}-\mathrm{H}_{2} \mathrm{O}$ interactions are insignificant in the electrolyte (low water concentration), the strength of the water-cation interaction directly impacts the $\mathrm{O}-\mathrm{H}$ bond strength and thus dictates the water reactivity upon reduction. By creating $\mathrm{H}$-bond 
interactions between water molecules at the short-range, small water clusters are formed which can impact the water reactivity only if the cation-water interaction is not too strong. Nevertheless, when the size of these clusters is grown by increasing the salt concentration, thus creating nanodomains in the electrolyte as detected by SAXS, an increase of the water reactivity is measured. Classical MD simulation shows that these structural heterogeneities are conserved at the interface. Overall, this confirms at the molecular scale that the transport of hydroxides and hydroniums ions at the electrode-electrolyte interface through several layers of water is a key parameter controlling the water reactivity.

Mastering the water structure at the electrode-electrolyte interface has recently been highlighted both from theoretica ${ }^{28,37}$ and experimental ${ }^{5,15,35}$ studies as critical to improve the catalytic activity of a surface toward water splitting. The framework developed in this work presents a molecular picture of how short and longranges interactions can be employed to promote water reduction on conductive electrodes in non-aqueous electrolytes. This acquired knowledge may transpose beyond the HER to other domains such as the electrosynthesis of complex organic molecule where it was for instance recently theoretically predicted that the formation of domains at the electrode-electrolyte interface may drastically influence electrochemical synthesis kinetics. ${ }^{38}$ Thus we believe that a similar approach that the one developed in this study may be employed to confirm these theoretical predictions. This picture may also been of interest to explain the decrease of potential window stability measured in wet ionic liquids, ${ }^{39-41}$ or explain the surprising poor cathodic stability of water-in-salt electrolytes that contains $\mathrm{Li}_{-} \mathrm{H}_{2} \mathrm{O}$ rich domains. ${ }^{42-46}$ Moreover, the creation of these kind of nanodomains in the electrolyte may also be used to tune the selectivity of reactions such as $\mathrm{CO}_{2}{ }^{47}$ or $\mathrm{N}_{2}{ }^{48}$ reduction by playing on the affinity of the reactants/products with an aqueous or hydrophobic nano-phase.

\section{References}

\section{The Future of Hydrogen, IEA, June 2019.}


2. Kibsgaard, J. \& Chorkendorff, I. Considerations for the scaling-up of water splitting catalysts. Nature Energy 4, 430-433 (2019).

3. Zheng, Y., Jiao, Y., Jaroniec, M. \& Qiao, S. Z. Advancing the Electrochemistry of the Hydrogen-Evolution Reaction through Combining Experiment and Theory. Angewandte Chemie International Edition 54, 52-65 (2015).

4. Zheng, Y., Jiao, Y., Vasileff, A. \& Qiao, S.-Z. The Hydrogen Evolution Reaction in Alkaline Solution: From Theory, Single Crystal Models, to Practical Electrocatalysts. Angewandte Chemie International Edition 57, 7568-7579 (2018).

5. Hu, C., Zhang, L. \& Gong, J. Recent progress made in the mechanism comprehension and design of electrocatalysts for alkaline water splitting. Energy \& Environ. Science 10.1039.C9EE01202H (2019) doi:10.1039/C9EE01202H.

6. Subbaraman, R. et al. Enhancing Hydrogen Evolution Activity in Water Splitting by Tailoring Li+-Ni(OH)2-Pt Interfaces. Science 334, 1256-1260 (2011).

7. Trasatti, S. Work function, electronegativity, and electrochemical behaviour of metals. Journal of Electroanalytical Chemistry and Interfacial Electrochemistry 39, 163-184 (1972).

8. Nørskov, J. K. et al. Trends in the Exchange Current for Hydrogen Evolution. Journal of The Electrochemical Society 152, J23 (2005).

9. Sheng, W. et al. Correlating hydrogen oxidation and evolution activity on platinum at different $\mathrm{pH}$ with measured hydrogen binding energy. Nature Communications 6, 5848 (2015).

10. Zheng, J., Sheng, W., Zhuang, Z., Xu, B. \& Yan, Y. Universal dependence of hydrogen oxidation and evolution reaction activity of platinum-group metals on $\mathrm{pH}$ and hydrogen binding energy. Science Advances 2, e1501602 (2016). 
11. Chen, X., McCrum, I. T., Schwarz, K. A., Janik, M. J. \& Koper, M. T. M. Co-adsorption of Cations as the Cause of the Apparent pH Dependence of Hydrogen Adsorption on a Stepped Platinum Single-Crystal Electrode. Angewandte Chemie International Edition 56, 15025-15029 (2017).

12. Janik, M. J., McCrum, I. T. \& Koper, M. T. M. On the presence of surface bound hydroxyl species on polycrystalline Pt electrodes in the "hydrogen potential region" (0-0.4 VRHE). Journal of Catalysis 367, 332-337 (2018).

13. Dubouis, N. \& Grimaud, A. J. L. The Hydrogen Evolution Reaction: From Material to Interfacial Descriptors. Chemical Science 10.1039.C9SC03831K (2019) doi:10.1039/C9SC03831K.

14. Velasco-Velez, J.-J. et al. The structure of interfacial water on gold electrodes studied by x-ray absorption spectroscopy. Science 346, 831-834 (2014).

15. Wu, C. H. et al. Molecular-Scale Structure of Electrode-Electrolyte Interfaces: The Case of Platinum in Aqueous Sulfuric Acid. Journal of the American Chemical Society 140, $16237-16244$ (2018).

16. Li, C.-Y. et al. In situ probing electrified interfacial water structures at atomically flat surfaces. Nature Materials 18, 697-701 (2019).

17. Ledezma-Yanez, I. et al. Interfacial water reorganization as a pH-dependent descriptor of the hydrogen evolution rate on platinum electrodes. Nature Energy 2, 17031 (2017).

18. Sarabia, F. J., Sebastián-Pascual, P., Koper, M. T. M., Climent, V. \& Feliu, J. M. Effect of the Interfacial Water Structure on the Hydrogen Evolution Reaction on $\mathrm{Pt}(111)$ Modified with Different Nickel Hydroxide Coverages in Alkaline Media. ACS Applied Materials \& Interfaces 11, 613-623 (2019). 
19. Dubouis, N. et al. The Fate of Water at the Electrochemical Interfaces: Electrochemical Behavior of Free Water Versus Coordinating Water. The Journal of Physical Chemistry Letters 9, 6683-6688 (2018).

20. Suárez-Herrera, M. F., Costa-Figueiredo, M. \& Feliu, J. M. Voltammetry of Basal Plane Platinum Electrodes in Acetonitrile Electrolytes: Effect of the Presence of Water. Langmuir 28, 5286-5294 (2012).

21. Ledezma-Yanez, I., Díaz-Morales, O., Figueiredo, M. C. \& Koper, M. T. M. Hydrogen Oxidation and Hydrogen Evolution on a Platinum Electrode in Acetonitrile.

ChemElectroChem 2, 1612-1622 (2015).

22. Ledezma-Yanez, I. \& Koper, M. T. M. Influence of water on the hydrogen evolution reaction on a gold electrode in acetonitrile solution. Journal of Electroanalytical Chemistry 793, 18-24 (2017).

23. Cassone, G., Creazzo, F., Giaquinta, P. V., Sponer, J. \& Saija, F. Ionic diffusion and proton transfer in aqueous solutions of alkali metal salts. Physical Chemistry Chemical Physics 19, 20420-20429 (2017).

24. Thordarson, P. Determining association constants from titration experiments in supramolecular chemistry. Chemical Society Review 40, 1305-1323 (2011).

25. Marx, D., Tuckerman, M. E., Hutter, J. \& Parrinello, M. The nature of the hydrated excess proton in water. Nature 397, 601-604 (1999).

26. Tuckerman, M. E., Marx, D. \& Parrinello, M. The nature and transport mechanism of hydrated hydroxide ions in aqueous solution. Nature 417, 925-929 (2002).

27. Geissler, P. L. Autoionization in Liquid Water. Science 291, 2121-2124 (2001). 
28. Filhol, J.-S. \& Neurock, M. Elucidation of the Electrochemical Activation of Water over Pd by First Principles. Angewante Chemie International Edition 45, 402-406 (2006).

29. Lange, K. M., Hodeck, K. F., Schade, U. \& Aziz, E. F. Nature of the Hydrogen Bond of Water in Solvents of Different Polarities. J. Phys. Chem. B 114, 16997-17001 (2010).

30. Lange, K. M. et al. On the Origin of the Hydrogen-Bond-Network Nature of Water: XRay Absorption and Emission Spectra of Water-Acetonitrile Mixtures. Angewandte Chemie International Edition 50, 10621-10625 (2011).

31. Huang, Y., Nielsen, R. J., Goddard, W. A. \& Soriaga, M. P. The Reaction Mechanism with Free Energy Barriers for Electrochemical Dihydrogen Evolution on MoS 2 . Journal of the American Chemical Society 137, 6692-6698 (2015).

32. Nishikawa, K., Kasahara, Y. \& Ichioka, T. Inhomogeneity of Mixing in Acetonitrile Aqueous Solution Studied by Small-Angle X-ray Scattering. Journal of Physical Chemistry B 106, 693-700 (2002).

33. Takamuku, T. et al. Large-Angle X-ray Scattering and Small-Angle Neutron Scattering Study on Phase Separation of Acetonitrile-Water Mixtures by Addition of NaCl. Journal of Physical Chemistry B 105, 6236-6245 (2001).

34. Huang, N. et al. X-ray Raman scattering provides evidence for interfacial acetonitrilewater dipole interactions in aqueous solutions. The Journal of Chemical Physics 135, 164509 (2011).

35. Brehm, M., Weber, H., Thomas, M., Hollóczki, O. \& Kirchner, B. Domain Analysis in Nanostructured Liquids: A Post-Molecular Dynamics Study at the Example of Ionic Liquids. ChemPhysChem 16, 3271-3277 (2015). 
36. Liu, E. et al. Unifying the Hydrogen Evolution and Oxidation Reactions Kinetics in Base by Identifying the Catalytic Roles of Hydroxyl-Water-Cation Adducts. Journal of the American Chemical Society 141, 3232-3239 (2019).

37. Chen, M. et al. Hydroxide diffuses slower than hydronium in water because its solvated structure inhibits correlated proton transfer. Nature Chemistry 10, 413-419 (2018).

38. Hollóczki, O., Macchieraldo, R., Gleede, B., Waldvogel, S. R. \& Kirchner, B. Interfacial Domain Formation Enhances Electrochemical Synthesis. Journal Physical Chemistry Letter 10, 1192-1197 (2019).

39. Meng, Y., Aldous, L., Belding, S. R. \& Compton, R. G. The hydrogen evolution reaction in a room temperature ionic liquid: mechanism and electrocatalyst trends. Physical Chemistry Chemical Physics 14, 5222-5228 (2012).

40. Bi, S. et al. Minimizing the electrosorption of water from humid ionic liquids on electrodes. Nature Communications 9, 5222 (2018).

41. Feng, G., Jiang, X., Qiao, R. \& Kornyshev, A. A. Water in Ionic Liquids at Electrified Interfaces: The Anatomy of Electrosorption. ACS Nano 8, 11685-11694 (2014).

42. Suo, L. et al. "Water-in-salt" electrolyte enables high-voltage aqueous lithium-ion chemistries. Science 350, 938-943 (2015).

43. Yamada, Y. et al. Hydrate-melt electrolytes for high-energy-density aqueous batteries. Nature Energy 1, 16129 (2016).

44. Borodin, O. et al. Liquid Structure with Nano-Heterogeneity Promotes Cationic Transport in Concentrated Electrolytes. ACS Nano 11, 10462-10471 (2017). 
45. Dubouis, N. et al. The role of the hydrogen evolution reaction in the solid-electrolyte interphase formation mechanism for "Water-in-Salt" electrolytes. Energy \& Environmental Science 11, 3491-3499 (2018).

46. McEldrew, M., Goodwin, Z. A. H., Kornyshev, A. A. \& Bazant, M. Z. Theory of the Double Layer in Water-in-Salt Electrolytes. Journal of Physical Chemistry Letter 9, 58405846 (2018).

47. Birdja, Y. Y. et al. Advances and challenges in understanding the electrocatalytic conversion of carbon dioxide to fuels. Nature Energy 4, 732-745 (2019).

48. Andersen, S. Z. et al. A rigorous electrochemical ammonia synthesis protocol with quantitative isotope measurements. Nature 570, 504-508 (2019).

\section{Acknowledgments}

N.D. acknowledges the Ecole normale supérieure for his PhD. scholarship. We acknowledge the French National Research Agency for its support through the Labex STORE-EX project (ANR-10LABX-76-01). This project has received funding from the European Research Council under the European Union's Horizon 2020 research and innovation program (Grant No. 771294). We acknowledge SOLEIL for provision of synchrotron radiation facilities and we would like to thank Thomas Bizien for assistance in using beamline SWING.

\section{Authors Contributions}

N.D. and A.G. designed the experiments. N.D. prepared and characterized the electrolytes. N.D. realized the electrochemical measurements and analyzed them with A.G. N.D. carried out the NMR characterization of 
the electrolytes, that were further analyzed with E.S. Diffusion NMR experiments were performed and analyzed by B.P. and E.S. N.D., A.S, R.B. and G.J. performed the MD simulations of the electrolytes and analyzed and designed them with M.S. All the authors edited the manuscript and discussed the scientific results.

\section{Competing interests}

The authors declare no competing interests

\section{Material and methods}

\section{General procedures}

All the experiments were carried out in an Ar-filled glovebox (MBraun, $\mathrm{O}_{2}<0.5 \mathrm{ppm} \mathrm{H}_{2} \mathrm{O}<0.5 \mathrm{ppm}$ ). All the salts were dried under vacuum for $24 \mathrm{~h}$ in a Büchi oven at $80^{\circ} \mathrm{C}$ and directly transferred to the glovebox. The electrolytes were prepared by adding a precise weight of Ar-flushed Milli-Q $\mathrm{H}_{2} \mathrm{O}$ to acetonitrile (Acros organics; $99.9 \%$, AcroSeal $^{\oplus}$, over molecular sieves) so that the final electrolyte contains the right weight percentage of $\mathrm{H}_{2} \mathrm{O}$. To these as-prepared solution, a precise mass of salt (anhydrous $\mathrm{LiClO}_{4}$ Alfa Aesar, 99\%, LiTFSI, Solvay, $\mathrm{NaClO}_{4}$ Alfa Aesar, Battery Grade or $\mathrm{TBAClO}_{4}$, Sigma-Aldrich for electrochemical analysis, 299.0\%) was added to obtain the desired cation/water ratio. The electrochemical cells were rinsed in aquaregia, boiled in Milli-Q water before to be rinsed 5 times with fresh Milli-Q and finally dried $1 \mathrm{~h}$ at $80^{\circ} \mathrm{C}$ prior to be used. The density of the electrolyte was measured by weighing $2.0 \pm 0.025 \mathrm{~mL}$ (volumetric flask) of each electrolyte. Their conductivity was measured with a SevenCompact 230 (Mettler Toledo) conductivitymeter. All their physical properties (density, concentration) are given in the SI.

\section{Electrochemical measurements}

Data were acquired on a Biologic VMP3 potentiostat. Prior to any measurement, platinum polycrystalline electrodes (5 mm diameter, Pine research) were polished using three polishing slurries (6 $\mu \mathrm{m}$ diamond on nylon polishing disk, followed by $0.3 \mu \mathrm{m}$ and $0.04 \mu \mathrm{m}$ aluminum oxide on microcloth polishing disk) using 
a polishing machine (Le Cube, Presi). Residual traces of slurries were removed by sonicating the as-polished electrode in a 50:50 $\mathrm{H}_{2} \mathrm{O}: \mathrm{EtOH}$ solution two times for 2 minutes. To ensure the cleanliness of the platinum electrodes surface, the electrodes were held at + $2.0 \mathrm{~V}$ vs. SHE in a $0.5 \mathrm{M} \mathrm{H}_{2} \mathrm{SO}_{4}$ solution purged with Ar for 2 minutes followed by 10 cycles of cyclic voltammetry (from $1.350 \mathrm{~V}$ to $0.0 \mathrm{~V}$ vs. SHE) in a fresh $\mathrm{H}_{2} \mathrm{SO}_{4}$ solution. Then, freshly cleaned electrodes were rinsed with ultrapure water, air-dried and pumped in the glovebox antechamber. Inside the glovebox, the electrodes were immediately covered by a drop of acetonitrile before being mounted onto a rotating disk setup (Pine research). All electrochemical measurements were recorded using a three electrodes cell setup with an $\mathrm{AgNO}_{3} / \mathrm{Ag}$ organic reference electrode (regularly calibrated against ferrocene). During the measurement, the working electrode was rotated at $1600 \mathrm{rpm}$. A flameannealed platinum wire was used as a counter electrode and placed in a separate compartment. A sweep rate of $50 \mathrm{mV} . \mathrm{s}^{-1}$ was used during all the electrochemical experiments. The ohmic drop was measured using current-interrupt technique after every electrochemical measurement. Typical values of around $\sim 50$ to 100 $\Omega$ were obtained. The ohmic drop compensation was performed during the data treatment (85\% of correction, as suggested by the potentiostat manufacturer).

\section{Nuclear-magnetic resonance characterization}

Liquid-state NMR spectra were recorded on a Bruker 7.046 T Avance III HD NMR spectrometer mounted with

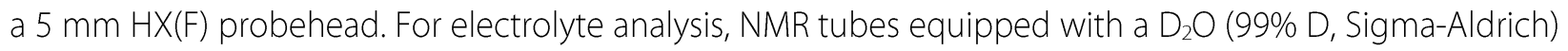

filled coaxial insert were used in order to lock the magnetic field and HOD signal was used as an internal reference (4.7 ppm). Single pulses sequences were used to record the ${ }^{1} \mathrm{H}$ and ${ }^{7} \mathrm{Li}$ spectra.

\section{${ }^{1} \mathrm{H}$ and ${ }^{7} \mathrm{Li}$ self-diffusion coefficient measurements by NMR}

The self-diffusion coefficients were measured using 10mm tubes in a Bruker Advance III HD 4.7 T operating at $200 \mathrm{MHz}$ for ${ }^{1} \mathrm{H}$ and $77.7 \mathrm{MHz}$ for ${ }^{7} \mathrm{Li}$, using a Bruker $10 \mathrm{~mm}$ liquid-state probe equipped with a ${ }^{1} \mathrm{H}$ or ${ }^{7} \mathrm{Li}$ $10 \mathrm{~mm}$ saddle coil. The pulsed magnetic field gradients (PFG) were performed with a Bruker diff30 pulsed magnetic field gradient (up to $30 \mathrm{G} . \mathrm{cm}^{-1} . \mathrm{A}^{-1}$ ) coil and a Bruker GREAT60 gradient amplifier. A stimulated 
echo ${ }^{49}$ was used with diffusion times of 7 to $10 \mathrm{~ms}$ and encoding/decoding square PFG pulses (1 to $2 \mathrm{~ms}$ ). The PFG amplitudes were varied in 32 steps from 0 to $600 \mathrm{G} / \mathrm{cm}\left({ }^{7} \mathrm{Li}\right)$ and from 0 to $300 \mathrm{G} / \mathrm{cm}\left({ }^{1} \mathrm{H}\right)$. A saturation, 2 dummy scans and a repetition time of $4 \mathrm{~s}$ for ${ }^{7} \mathrm{Li}$ and $5 \mathrm{~s}$ for ${ }^{1} \mathrm{H}$ were used to ensure steady state. At least 32 transients were added for each measurement.

The Bruker Control Unit (BCU20) was used to set 5 temperatures for the water in the PFG coil (281 K to $321 \mathrm{~K}$ in steps of $10 \mathrm{~K}$ ), and the temperature was calibrated using a $10 \mathrm{~mm}$ tube filled with glycerol in the same conditions. The sample was left to equilibrate for at least 30 minutes in the spectrometer before the measurements. The data processing is described in the Supplementary information.

\section{Small-angle X-Ray Scattering}

Small-angle X-ray scattering (SAXS) data were collected at the SWING beamline (SOLEIL synchrotron, SaintAubin, France). The beamline was operated at $16 \mathrm{keV}$ and an Eiger 4M Detector (Dectris) was used. Electrolyte samples were filled into $1 \mathrm{~mm}$ capillaries inside the glovebox, sealed with epoxy and the measurements were performed at room temperature. The sample-to-detector distances were set to $6.2 \mathrm{~m}$ and $0.5 \mathrm{~m}$, which allowed to cover scattering vectors in the range from $q=0.0016 \AA^{-1}$ to $0.28 \AA^{-1}$. The data analysis (including integration, normalization and background subtraction) was carried out using the FOXTROT software.

\section{Bulk Molecular Dynamic Simulations}

Classical MD simulations of the bulk systems were performed using the GROMACS software package.50 Acetonitrile solvent was described with a six-site model. ${ }^{51}$ SPC/E model was chosen for water molecules. ${ }^{52}$ Force field parameters for perchlorate anions and TBA cations were taken from Ref. 53 and Ref. 54, respectively. The Lennard-Jones parameters for $\mathrm{Li}^{+}$and $\mathrm{Na}^{+}$were taken from Åqvist. ${ }^{55}$ Mixed Lennard-Jones parameters for all of the different atom types were obtained from the Lorentz-Berthelot combination rules. The simulations were performed in the NVT ensemble at $300 \mathrm{~K}$ for $100 \mathrm{~ns}$, with the Nosé-Hoover thermostat ${ }^{56,57}$ (the relaxation constant used is 0.5 ps), using a timestep of $1 \mathrm{fs}$ and saving a configuration 
every $100 \mathrm{fs}$. The initial configuration was achieved by generating a low density cubic box by means of the PACKMOL package, ${ }^{58}$ that was then compressed in the NPT ensemble in order to reproduce the electrolyte density at temperature and pressure conditions of $300 \mathrm{~K}$ and $1 \mathrm{~atm}$, respectively. All the box configurations are given in Supplementary Tables 1-3. The systems were then equilibrated in the NVT ensemble at $700 \mathrm{~K}$ for $0.5 \mathrm{~ns}$, followed by a final NVT equilibration at $300 \mathrm{~K}$ for $2 \mathrm{~ns}$. The production simulations were finally carried out in the NVT ensemble at $300 \mathrm{~K}$ for $100 \mathrm{~ns}$. Long-range electrostatic interactions were computed with the particle mesh Ewald method, ${ }^{59}$ while a cutoff distance of $9 \AA$ was adopted for the non-bonded interactions. The LINCS algorithm was employed to constrain the stretching interactions involving hydrogen atoms. ${ }^{60}$ The coordination numbers were obtained by the integration of the radial distribution functions (RDF), calculated every 100 fs using the GROMACS utilities..$^{50}$ The structure factors were calculated with an in-house code using the partial RDFs. The domain analysis was performed using TRAVIS, ${ }^{35,61}$ while VMD software was used to obtain the snapshots. ${ }^{62}$

\section{Molecular Dynamic Simulations at constant applied voltage}

Classical MD simulations of the electrolytes containing $\mathrm{LiClO}_{4}$ between two planar graphite electrodes were performed at a fixed potential difference of $1 \mathrm{~V}$ using an in-house code, following the approach detailed in Refs. $^{63,64} 2 \mathrm{D}$ periodic boundary conditions were used in the xy directions. The force field parameters were kept the same as in the bulk simulations, with the addition of the Lennard-Jones parameters for the electrode carbon atoms taken from Ref.65 The simulations were performed in the NVT ensemble at $300 \mathrm{~K}$, with the Nosé-Hoover thermostat ${ }^{56,57}$ (the relaxation constant used is 1 ps), using a timestep of $1 \mathrm{fs}$. Long-range electrostatic interactions were computed with the Ewald summation method in 2D with a combination of point charges for the electrolyte and Gaussian charges for the electrodes, ${ }^{63,66}$ while a cutoff distance of $12 \AA$ was adopted for the non-bonded interactions. The SHAKE algorithm was employed to constrain the stretching interactions involving hydrogen atoms. ${ }^{67,68}$ Further details about the box sizes and geometries are provided in Supplementary Table 4. The systems were first pre-equilibrated by fixing the electrode 
charges to zero (about $400 \mathrm{ps}$ ) and then under constant applied potential of $\mathrm{IV}$. The equilibration time was

$2.5 \mathrm{~ns}, 1.5 \mathrm{~ns}$ and $3.0 \mathrm{~ns}$ for the three systems, respectively. Once the steady state was reached, the systems were simulated for $5.2 \mathrm{~ns}, 3.0 \mathrm{~ns}$ and $3.7 \mathrm{~ns}$, respectively.

\section{Data availability}

The data related to this study are available from the authors upon reasonable request.

\section{Material and Methods References}

49. Tanner, J. E. Use of the Stimulated Echo in NMR Diffusion Studies. Journal of Chemical Physics 52, 2523-2526 (1970).

50. Abraham, M. J. et al. GROMACS: High performance molecular simulations through multi-level parallelism from laptops to supercomputers. SoftwareX 1-2, 19-25 (2015).

51. Grabuleda, X., Jaime, C. \& Kollman, P. A. Molecular dynamics simulation studies of liquid acetonitrile: New six-site model. Journal of Computational Chemistry 21, 901-908 (2000).

52. Berendsen, H. J. C., Grigera, J. R. \& Straatsma, T. P. The missing term in effective pair potentials. Journal of Physical Chemistry 91, 6269-6271 (1987).

53. Liu, X. et al. New Force Field for Molecular Simulation of Guanidinium-Based Ionic Liquids. Journal of Physical Chemistry B 110, 12062-12071 (2006).

54. Bhowmik, D. et al. Aqueous solutions of tetraalkylammonium halides: ion hydration, dynamics and ion-ion interactions in light of steric effects. Physical Chemistry Chemical Physics 16, 13447-13457 (2014). 
55. Ȧqvist, J. Ion-water interaction potentials derived from free energy perturbation simulations. Journal of Physical Chemistry 94, 8021-8024 (1990).

56. Nosé, S. A unified formulation of the constant temperature molecular dynamics methods. Journal of Chemical Physics. 81, 511-519 (1984).

57. Evans, D. J. \& Holian, B. L. The Nose-Hoover thermostat. Journal of Chemical Physics 83, 4069-4074 (1985).

58. Martínez, L., Andrade, R., Birgin, E. G. \& Martínez, J. M. PACKMOL: A package for building initial configurations for molecular dynamics simulations. Journal of Computational Chemistry 30, 2157-2164 (2009).

59. Essmann, U. et al. A smooth particle mesh Ewald method. Journal of Chemical Physics. 103, 8577-8593 (1995).

60. Hess, B., Bekker, H., Berendsen, H. J. C. \& Fraaije, J. G. E. M. LINCS: A linear constraint solver for molecular simulations. Journal of Computational Chemistry 18, 14631472 (1997).

61. Brehm, M. \& Kirchner, B. TRAVIS - A Free Analyzer and Visualizer for Monte Carlo and Molecular Dynamics Trajectories. Journal of Chemical Information and Modeling 51, 2007-2023 (2011).

62. Humphrey, W., Dalke, A. \& Schulten, K. VMD: Visual molecular dynamics. Journal of Molecular Graphics 14, 33-38 (1996).

63. Reed, S. K., Lanning, O. J. \& Madden, P. A. Electrochemical interface between an ionic liquid and a model metallic electrode. Journal of Chemical Physics 126, 084704 (2007).

64. Siepmann, J. I. \& Sprik, M. Influence of surface topology and electrostatic potential on water/electrode systems. Journal of Chemical Physics 102, 511-524 (1995). 
65. Werder, T., Walther, J. H., Jaffe, R. L., Halicioglu, T. \& Koumoutsakos, P. On the Water-Carbon Interaction for Use in Molecular Dynamics Simulations of Graphite and Carbon Nanotubes. Journal of Physical Chemistry B 107, 1345-1352 (2003).

66. Gingrich, T. R. \& Wilson, M. On the Ewald summation of Gaussian charges for the simulation of metallic surfaces. Chemical Physics Letters 500, 178-183 (2010).

67. Ryckaert, J.-P., Ciccotti, G. \& Berendsen, H. J. C. Numerical integration of the cartesian equations of motion of a system with constraints: molecular dynamics of n-alkanes. Journal of Computational Physics 23, 327-341 (1977).

68. Ciccotti, G., Ferrario, M. \& Ryckaert, J.-P. Molecular dynamics of rigid systems in cartesian coordinates A general formulation. Molecular Physics 47, 1253-1264 (1982). 